\title{
Incidentally Solitary, Synchronous, Metastatic Left Adrenal Mass From Colon Cancer
}

\author{
Mina Alvandipour, Mohammad Yasin Karami ${ }^{1}$, Mehdi Khalvati, Hamed Khodabakhsh ${ }^{2}$ \\ Department of Surgery, Mazandaran University of Medical Sciences, Sari; ${ }^{1}$ Department of Surgery, Student Research Committee, Shiraz \\ University of Medical Sciences, Shiraz; ${ }^{2}$ Department of Emergency Medicine, Faculty of Medicine, Mazandaran University of Medical \\ Sciences, Sari, Iran
}

The authors report the case of a 63-year-old man who underwent an open adrenalectomy for a synchronous, malignant, metastatic left adrenal tumor and a total colectomy for T3N0M1 (stage 4) primary, malignant colon cancer. Two polypoid lesions, one measuring $40 \mathrm{~mm} \times 30 \mathrm{~mm} \times 30 \mathrm{~mm}$ and the other measuring $20 \mathrm{~mm} \times 10 \mathrm{~mm} \times 10 \mathrm{~mm}$, were found in the ascending colon and rectosigmoid (RS) junction, respectively, and a synchronous, malignant, left adrenal gland lesion measuring $70 \mathrm{~mm} \times 50 \mathrm{~mm} \times 30 \mathrm{~mm}$ was incidentally found on abdominal computed tomography scan. Histological examination revealed a metastatic, necrotic adenocarcinoma of the left adrenal mass, an adenocarcinoma of the cecal mass, and an adenomatous polyp (tubulovillous type) of the smallest polypoid lesion in RS junction that had invaded deeply into the submucosal layer. The patient recovered uneventfully, and his condition is now stable, with no evidence of local recurrence or metastatic disease, 2 years after the surgery. To the best of our knowledge, only 25 cases of an adrenalectomy for treating metastatic adrenal gland tumors have been reported to date; physicians should be aware of the possibility of this event.

Keywords: Colonic neoplasms; Adrenal gland neoplasms; Adrenalectomy; Colectomy; Multiple primary

\section{INTRODUCTION}

Adrenal metastasis most commonly occurs in patients with lung and breast cancer, and its metastasis from colorectal cancer (CRC) has generally been established to be rare $[1,2]$. Adrenal metastases are considered to be the result of a haematogenous spread of the primary carcinoma from CRC and to be unsuitable for surgical resection. Surgical treatment of the implicated adrenal gland did not seem not to offer a survival advantage for this patient while chemoradiotherapy had poor results [2]. The case of a patient who underwent an open resection of a synchronous left adrenal metastasis from colon cancer and a total colectomy at the

Received: December 25, 2015 - Accepted: February 6, 2016

Correspondence to: Mohammad Yasin Karami, M.D.

Department of Surgery, Faghihi Hospital, Shiraz University of Medical

Sciences, Shiraz, Iran

Tel: +98-9171800710, Fax: +98-7132330724

E-mail:karamiy@sums.ac.ir

(c) 2016 The Korean Society of Coloproctology

This is an open-access article distributed under the terms of the Creative Commons Attribution NonCommercial License (http://creativecommons.org/licenses/by-nc/4.0) which permits unrestricted noncommercial use, distribution, and reproduction in any medium, provided the original work is properly cited. same time is reported. This case is an unusual case of left adrenal metastasis occurring during colon-cancer management, which had been successful.

\section{CASE REPORT}

A 63-year-old man, known to be on oral medication for diabetes mellitus, was referred to the Colorectal Clinic of Academic Hospital (Emam Khomeini Hospital) with complaints of having had abdominal pain, rectoragy and constipation during the four months prior to the visit. The patient stated that the pain in the right lower quadrant of the abdomen had started gradually and radiated to the epigastric area, finally changing to a constant, non-positionspecific pain one month earlier. The patient had lost $10 \mathrm{~kg}$ of weight during a short time (3 months) and had experienced gross rectal bleeding and constipation during that same period. Upon physical examination, the patient had normal vital signs and no sign of lymphadenopathy; chest, lung and heart examinations were normal. However, an abdominal examination revealed a soft, tender right lower quadrant with no palpable mass; the digital rectal examination was normal. The patient had no symptoms such as sweating, headache, paroxysmal hypertension, arrhythmias, 
palpitations, central obesity, thin extremities, supraclavicular fat, proximal muscle weakness, moon face, hypertension, buffalo hump, hyperglycemia, abdominal striae, metabolic alkalosis, skin thinning, hypokalemia, easy bruising, osteopenia, and poor wound healing. Laboratory testing showed a normal white blood count, normal differential counts and anemia (hemoglobin, $7 \mathrm{~g} /$ dL). Fasting blood sugar was $127 \mathrm{mg} / \mathrm{dL}$, serum creatinin was 0.7 $\mathrm{mg} / \mathrm{dL}$, and serum electrolytes were normal. Liver function tests were normal, as were other laboratory tests, including electrolytes; the results from the urinalysis were normal. The serum carcinoembryonic antigen (CEA) level was increased to $33.5 \mathrm{ng} / \mathrm{mL}$ (normal value, $<5 \mathrm{ng} / \mathrm{mL}$ ). Colonoscopy had shown one large pedunculated polyp in the RS junction and another giant circumferential tumor in the ascending colon. A polypectomy and a biopsy were performed, and the histopathology report confirmed the presence of both a moderately differentiated adenocarcinoma arising in a tubulo-villous adenoma of the RS junction mass with invasion into the deep submucosal layer and an adenocarcinoma of the ascending colon mass (Fig. 1). The presence of a solitary lesion in the left adrenal gland measuring $70 \mathrm{~mm} \times 50 \mathrm{~mm} \times 30 \mathrm{~mm}$ was incidentally detected on an abdominal and pelvic computed tomography (CT) scan (Fig. 2). Laboratory testing showed normal urine vanillylmandelic acid and serum metanephrine excretion. Therefore, an open left adrenalectomy and a total colectomy were performed simultaneously (Fig. 3).

Exploration of the abdomen showed the presences of no other metastatic lesions. Postoperative histological examination revealed a metastatic necrotic adenocarcinoma of the left adrenal mass (Fig. 4; yellowish, necrotic and soft, neoplastic-like tissue, with a definitive histology classified as adrenal metastasis from an intestinal adenocarcinoma), a moderately differentiated adenocarcinoma of the colon mass, and an adenomatous polyp (tubulo-
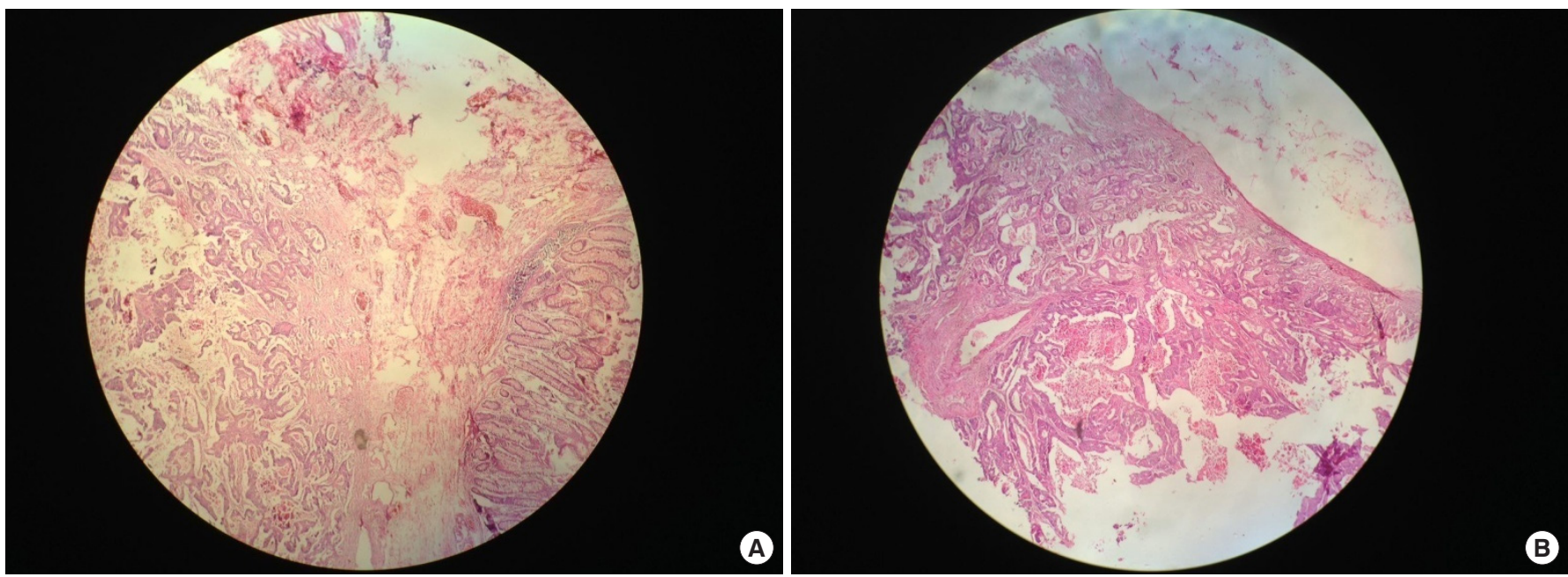

Fig. 1. (A) Moderately differentiated adenocarcinoma arising in a tubulo-villous adenoma of the rectosigmoid junction with invasion into the deep submucosal layer $(\mathrm{H} \& \mathrm{E}, \times 100)$. (B) Moderately differentiated adenocarcinoma of the ascending colon according to the mass's pathology $(\mathrm{H} \& \mathrm{E}, \times 100)$.
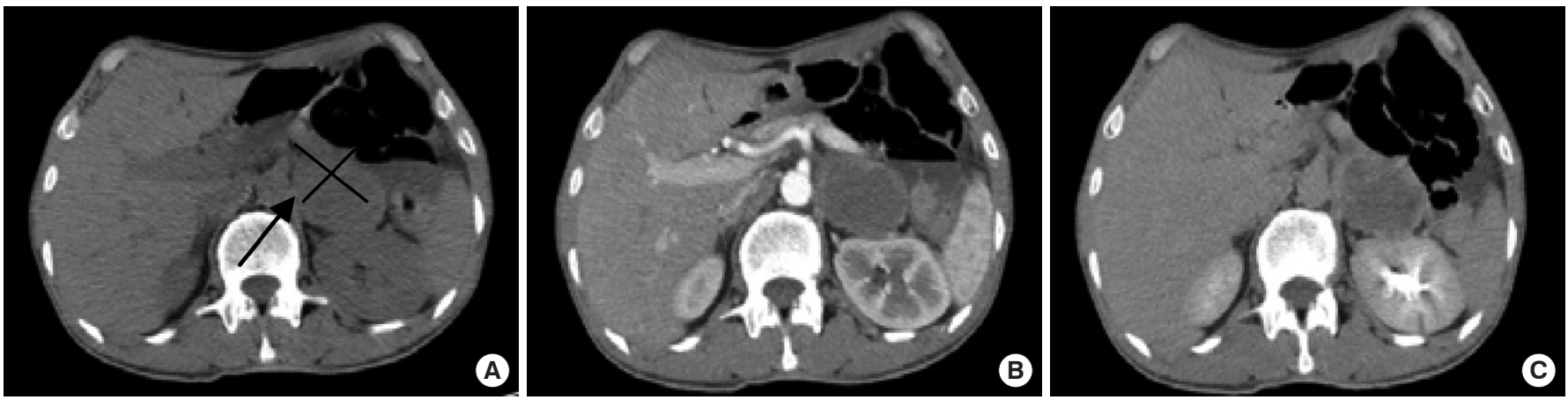

Fig. 2. (A) An abdominal computed tomogram demonstrated a $70 \times 50 \times 30$-mm mass in the left adrenal gland (The Black arrow showed left adrenal mass). (B) An early-phase dynamic adrenal gland computed tomography (CT) demonstrated a nonenhanced left adrenal mass. (C) A late-phase dynamic adrenal gland CT demonstrated a nonenhanced left adrenal mass. 


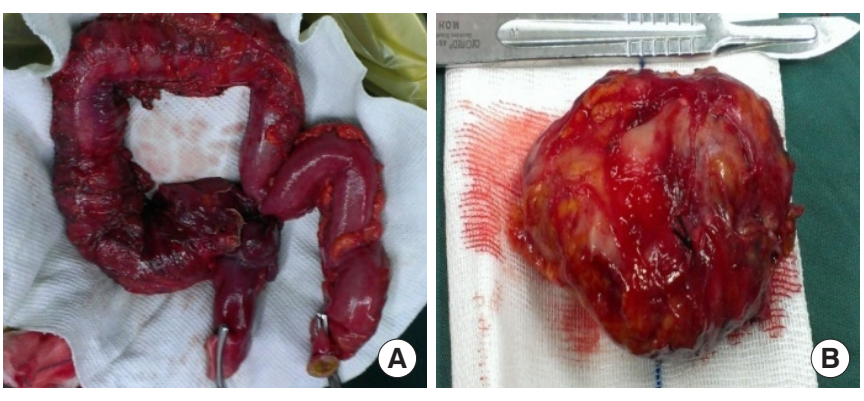

Fig. 3. (A) Total colectomy product. The black arrow points to the mass in the ascending colon. (B) Postoperative aspect of the left-side adrenal tumor.

villous type) of the smallest polypoid lesion in the RS junction with invasion into the deep mucosal layer. A cycle of adjuvant chemotherapy with FOLFOX (folinic acid, fluorouracil and oxaliplatin) was administrated. The patient was enrolled in a follow-up program, in which the serum CEA level would be determined at each follow-up visit for at least the first three years and CT of the chest, abdomen, and pelvis would be performed annually for at least three years. The postoperative course of the patient is still stable 2 years after surgery, and no evidence of local recurrence or metastatic disease has been seen. At the time of this report, the patient has survived for 48 months and is asymptomatic and in stable condition. Written informed consent was obtained from the patient for publication of this case report and accompanying images. A copy of the written consent is available for review from the Editor-in-Chief of this journal.

\section{DISCUSSION}

The most common sites of colorectal metastasis are the liver and the lungs. An adrenal metastasis from a colorectal carcinoma is not a rare event, and its incidence ranges from $1.9 \%$ to $17.4 \%$ in autopsy studies [3-6]. The most common primary tumors responsible for adrenal metastasis are breast, lung and kidney carcinomas. Adrenal metastasis from CRC has been suggested to proceed via a haematogenous route [7], and a curative resection of a adrenal metastasis from CRC is possible [8-10]. According to conventional wisdom, a lone adrenal metastasis should be resected to achieve a good prognosis, even though the incidence of such a resectable mass is low $[11,12]$. The use of a laparoscopic adrenalectomy for the treatment of large, malignant adrenal tumors is a subject of controversy [13].

The possibility of adrenal metastasis is important to consider in patients who have CRC. Although the prognosis for a patient with adrenal metastasis is poor, the adrenal metastasis should be completely removed if a better prognosis is to be achieved. Radiological modalities, such as ultrasonography (US) and CT, as well as the serum CEA level, are commonly used for the early detection of adrenal metastasis.

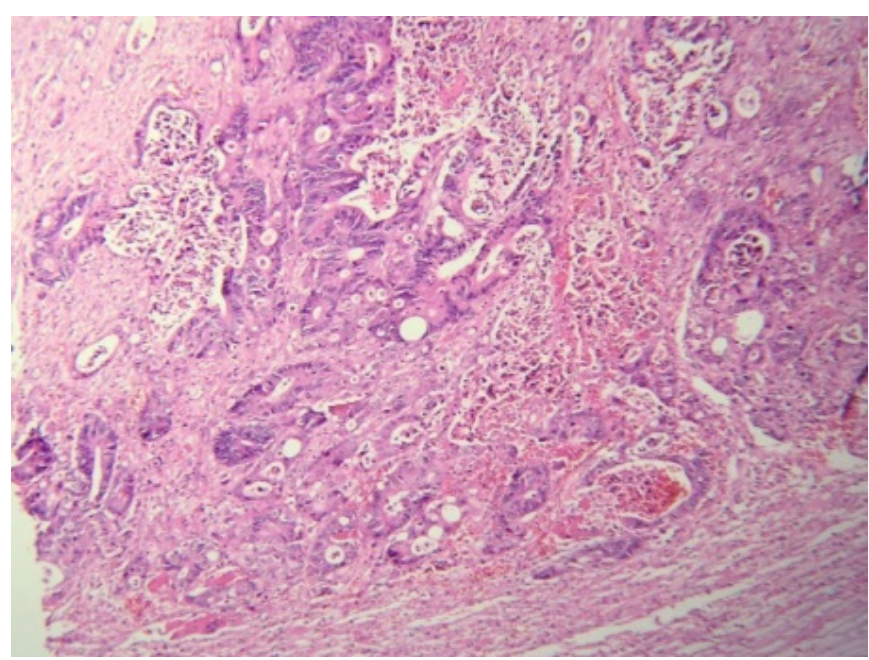

Fig. 4. Phathologic finding of metastatic adrenal cancer $(H \& E, \times 100)$.

In the majority of cases, with an 18-month median follow-up (range, 1-108 months), the average postoperative survival is about 21 months, with a 5 -year survival rate of $24 \%$, and the average disease-free survival rate is $11 \%$, with a 5 -year survival rate of $21 \%[14]$.

Adrenal metastasis from CRC is not rare; however, a solitary synchronous metastasis is extremely rarely. A left-sided adrenal mass with inferior vena cava (IVC) tumor thrombus is an uncommon presentation for this malignancy. As surgical (laparoscopic or open) treatment of the metastatic lesion could advance a patient's prognosis, the possibility of synchronous adrenal metastasis should be kept in mind when CRC patients are being diagnosed. Moreover, no surgical treatment that can be safely performed by an experienced surgeon should be overlooked. We suggest neoadjuvant chemotherapy for patients with stage- 3 and -4 colon cancer who have adrenal or other organ metastasis, especially those who do not present with anemia, Gastrointestinal bleeding or obstruction. Fine needle aspiration (FNA) or radiologically confirmed synchronous adrenal metastases could resected in laparoscopic or open procedure. Neoadjuvant chemotherapy and salvage surgery is recommended if the adrenal mass has invaded adjuvant organs or IVC thrombus has been.

In conclusion, the presence of a left-side, synchronous, solitary adrenal metastasis from CRC, as described in our case, is either very rare [15] or such cases have gone unreported. For early detection of an adrenal metastasis, preoperative radiological modalities, such as US and CT, as well as the determination of the serum CEA level, should normally be done. Definitely, a follow-up program, including a measurement of the CEA level at each followup visit for at least the first three years and annual CT scans of the chest, abdomen, and pelvis for at least three years, is needed.

In conclusion, for a better prognosis, the authors suggest the use of an open adrenalectomy for treating patients with CRC who 
were found during a colectomy to have malignant-like large adrenal metastasis. To the best of our knowledge, this is the first report of synchronous, left adrenal cancer metastasizing from colon cancer. This rare event has not been described previously, so colorectal surgeons should be aware of the possibility of this complication. The authors suggest that synchronous, metastatic left adrenal mass from colon cancer should be compared with other similar experiences.

\section{CONFLICT OF INTEREST}

No potential conflict of interest relevant to this article was reported.

\section{ACKNOWLEDGMENTS}

The authors would like to thank the patient and his wife for their cooperation. We also appreciate the copy editing by Dr. Mehrnoosh Maalhagh.

\section{REFERENCES}

1. Murakami S, Terakado M, Hashimoto T, Tsuji Y, Okubo K, Hirayama R. Adrenal metastasis from rectal cancer: report of a case. Surg Today 2003;33:126-30.

2. Kanjo T, Albertini M, Weber S. Long-term disease-free survival after adrenalectomy for isolated colorectal metastases. Asian J Surg 2006;29:291-3.

3. Glomset DA. The incidence of metastasis of malignant tumors to the adrenals. Am J Cancer 1938;32:57-61.

4. Abrams HL, Spiro R, Goldstein N. Metastases in carcinoma; analysis of 1000 autopsied cases. Cancer 1950;3:74-85.

5. Berge T, Ekelund G, Mellner C, Pihl B, Wenckert A. Carcinoma of the colon and rectum in a defined population. An epidemiological, clinical and postmortem investigation of colorectal carci- noma and coexisting benign polyps in Malmö, Sweden. Acta Chir Scand Suppl 1973;438:1-86.

6. Cedermark BJ, Blumenson LE, Pickren JW, Holyoke DE, Elias EG. Ths significance of metastases to the adrenal glands in adenocarcinoma of the colon and rectum. Surg Gynecol Obstet 1977; 144:537-46

7. Capaldi M, Ricci G, Bertolini R, Alessandroni L, Di Castro A, Saraco E, et al. Colon cancer adrenal metastasis: case report and review of the literature. G Chir 2011;32:361-3.

8. Watatani M, Ooshima M, Wada T, Terashita H, Matsuda T, Shindo $\mathrm{K}$, et al. Adrenal metastasis from carcinoma of the colon and rectum: a report of three cases. Surg Today 1993;23:444-8.

9. Katayama A, Mafune K, Makuuchi M. Adrenalectomy for solitary adrenal metastasis from colorectal carcinoma. Jpn J Clin Oncol 2000;30:414-6.

10. Fujita K, Kameyama S, Kawamura M. Surgically removed adrenal metastasis from cancer of the rectum: report of a case. Dis Colon Rectum 1988;31:141-3.

11. Masuyama K, Shimada K, Uotani H, Tajika S, Tsuji M, Matsunou H. A case of rectal cancer with metastasis to left adrenal cortical carcinoma. Nihon Geka Gakkai Zasshi 2001;62:192-6.

12. Yamada T, Egami K, Iida N, Nakamura Y, Teramoto T, Onda M. A resected case of adrenal metastasis from colon cancer. Shujutsu 2001;55:1421-5.

13. Zografos GN, Markou A, Ageli C, Kopanakis N, Koutmos S, Kaltsas G, et al. Laparoscopic surgery for adrenal tumors. A retrospective analysis. Hormones (Athens) 2006;5:52-6.

14. Kim SH, Brennan MF, Russo P, Burt ME, Coit DG. The role of surgery in the treatment of clinically isolated adrenal metastasis. Cancer 1998;82:389-94.

15. Komuro Y, Watanabe T, Kanazawa T, Tada T, Fukatsu K, Hosaka A, et al. A case of adrenal metastasis from rectal carcinoma without lung metastasis: evaluation for alteration of loss of heterozygosity and immunohistochemical expression. J Gastroenterol 2004;39:594-5. 